\title{
Discreteness in particle masses and parameters of the Standard Model
}

\section{Sergey Sukhoruchkin*}

B.P. Konstantinov Petersburg Nuclear Physics Institute, 188300 Gatchina, Russia

E-mail: sergeis@pnpi.spb.ru

\begin{abstract}
We performed simultaneous analysis of the discreteness in particle mass spectrum and relations in nuclear parameters connected with nuclear tensor force (one-meson exchange dynamics). Analysis of particle masses is in line with the suggestion that empirical relations in masses could be used for the development of the Standard Model. Due to a closeness of the pion mass splitting $\delta m_{\pi}=4594 \mathrm{keV}$ to the $9 m_{e}=4599 \mathrm{keV}$ ( $m_{e}$ is the electron mass) the parameter of the discreteness in particle mass spectrum is close to the doubled value of the pion $\beta$-decay energy $16 m_{e}=\delta \approx 2 \delta m_{\pi}-2 m_{e}$ (tuning effect in particle masses). This discreteness was found also in parameters of correlations in nuclear excitations and binding energies. Common tuning effect includes also the nucleon mass splitting $\delta m_{N}=1293.3 \mathrm{keV}$ which belongs to the fundamental manifestation of the nucleon structure. The value $\delta m_{N}$ and its $1 / 8$ part $\delta m_{N} / 8=161 \mathrm{keV}$ were found also in the grouping effect in nuclear excitations and spacing. In the tuning effect in particle masses the value (1/8) $\delta m_{N}=161 \mathrm{keV}$ accounts the shift of the exactly known neutron mass $m_{n}$ relative to integer number of the electron rest mass (so-called CODATA relation). We consider these correlations, the possible role of the lepton ratio and the possible hints on the symmetry in the fermion spectra as the new approach to the SM-development.
\end{abstract}

The European Physical Society Conference on High Energy Physics

22-29 July 2015

Vienna, Austria

${ }^{*}$ Speaker. 


\section{Introduction}

Nucleon interactions within nuclei are commonly considered as a result of one- and many-pion exchange. The role of tensor forces (one-pion exchange) was recently considered by J.Schiffer [1], T.Otsuka [2] and I.Tanihata [3]. They found the regions of nuclear chart where tensor-force effects are important. For example, in heavy antimony isotopes $(\mathrm{N}=72-82)$ the valence proton in $J^{\pi}=7 / 2^{+}$ state $\left(\pi, 1 g_{7 / 2}\right)$ strongly interacts with neutrons in the large $v, 1 h_{11 / 2}$ subshell. Such deuteronlike situation (spin 1, orbital motion in different directions) with the dominant role of one-pion exchange dynamics in nucleon interaction exists also in light and heavy nuclei. Stable intervals in first excitations of the near-magic ${ }^{116} \mathrm{Sn}\left(E^{*}=1293.6 \mathrm{keV}, E_{2}-E_{1}=1292.0 \mathrm{kev}\right)$ correspond to $\mathrm{n}=8$ in this systematics. They are close to the nucleon mass splitting $\delta m_{N}=1293.3 \mathrm{keV}$. The system $E^{*}, D$ $=\mathrm{n} \Delta^{T F}$ was confirmed with stable excitations and spacing in many nearby nuclei [4-8].

The linear trend in excitations $E^{*}$ of ${ }^{\text {odd }} \mathrm{Sb}$ isotopes with a slope $161 \mathrm{keV}, E^{*}=\mathrm{n}\left(\Delta^{T F}=161 \mathrm{keV}\right)$ is similar to that found in the first excitations of $\mathrm{N}=21,22$ isotopes ${ }^{41} \mathrm{Ca}$ and ${ }^{38,37} \mathrm{~S}$. Here $E^{*}=1942.8$ $1292.0-646.2 \mathrm{keV}$ coincide within $2-3 \mathrm{keV}$ with $\mathrm{n}=12,8,4$ in the system $\mathrm{n} \delta^{T F}$. In $\mathrm{Z}=72,74$ isotopes $E^{*}=1293-1293-1293-1295 \mathrm{keV}\left(0^{+}, 2^{+}\right.$in $\left.{ }^{160} \mathrm{Yb},{ }^{172,176} \mathrm{Hf},{ }^{178} \mathrm{~W}\right)$ correspond to $\mathrm{n}=8$. These $E^{*}, D$ and small intervals at high excitation with $\mathrm{D}$ close to $E^{*}(\alpha / 2 \pi)$ are presented in Table 1 (bottom).

The stable character of excitation about $170 \mathrm{keV}$ in the near-magic ${ }^{101,103} \mathrm{Sn}(\mathrm{N}=51,53)$ was confirmed with stable spacing of ${ }^{97,98} \mathrm{Pd}(\mathrm{N}=51,52)$. Here stable intervals $\mathrm{D}=512 \mathrm{keV}$ (close to $3 \times 170 \mathrm{keV}$, that's n'=3) and $\mathrm{D}=648 \mathrm{keV}$ and $1293 \mathrm{keV}\left(\mathrm{N}=4,8\right.$ in the system with $\left.\Delta^{T M}\right)$ was found. The latter interval corresponds to the excitations with $\Delta J=2,1295 \mathrm{keV}$ and $2588 \mathrm{keV})$. In ${ }^{10} \mathrm{~B}$ the interval $1021.72(3) \mathrm{keV}\left(\mathrm{n}^{\prime}=6\right)$ is a result of spin-flip effect $\left(0^{+}-1^{+}\right)$. Its value is close to $2 m_{e}=1022.0 \mathrm{keV}=\varepsilon_{o}$. Excitations $\mathrm{k} \cdot \varepsilon_{o}$ were noticed in many light nuclei including the magic ${ }^{55} \mathrm{Co}$. Similar effects with $\mathrm{n} \cdot 161 \mathrm{keV}=(1 / 8) \delta m_{N}$ and $\mathrm{n}^{\prime} \cdot 170 \mathrm{keV}=(1 / 6) \varepsilon_{o}$ are considered in [4-9].

Table 1. Presentation of particle masses and parameters from analysis of nuclear data (in lines marked $\mathrm{X}=-1,0,1,2$ at left) by the common expression $\mathrm{n} \cdot 16 \mathrm{~m}_{\mathrm{e}}(\alpha / 2 \pi)^{X} \mathrm{M}$ with the QED radiative correction $\alpha / 2 \pi$ $\left(\alpha=137^{-1}\right)[9,10]$. Boxed are values $M_{Z}$, scalar boson mass $M_{H}, m_{\mu}, \mathrm{m}_{\pi}-\mathrm{m}_{\mathrm{e}}, \mathrm{m}_{\mathrm{e}} / 3$, the neutron mass shift $\underline{\mathrm{N} \delta-m_{\mathrm{n}}-m_{\mathrm{e}} \approx \Delta^{T F} \text { and } \Delta M_{\Delta} \approx m_{s}}$. Stable $\mathrm{E}^{*}, D_{i j}$ (at X=1,2) indirectly confirm values at $\mathrm{X}=1$ (center).

\begin{tabular}{|c|c|c|c|c|c|c|c|}
\hline $\mathrm{X}$ & M & $\mathrm{n}=1$ & $\mathrm{n}=13$ & $\mathrm{n}=14$ & $n=16$ & $\mathrm{n}=17$ & $\mathrm{n}=18$ \\
\hline-1 & $3 / 2$ & & & & $\mathrm{~m}_{\mathrm{t}}=171.2$ & & \\
\hline $\mathrm{GeV}$ & 1 & $2 \Delta^{\circ}-2 \mathrm{M}_{\mathrm{q}}$ & $\mathrm{M}_{\mathrm{Z}}=91.2$ & & $\left(M_{\mathrm{H}}=115\right)$ & & $M_{\mathrm{H}}=125.7[14]$ \\
\hline 0 & 1 & $16 \mathrm{~m}_{\mathrm{e}}=\delta=8 \varepsilon_{o}$ & $\mathrm{~m}_{\mu}=105.7$ & & $\mathrm{f}_{\pi}=130.7$ & $\mathrm{~m}_{\pi}-\mathrm{m}_{\mathrm{e}}$ & $\mathrm{m}_{\Delta}-\mathrm{m}_{\mathrm{N}} / 2=147$ \\
\hline \multirow[t]{2}{*}{$\mathrm{MeV}$} & 3 & & & & $\mathrm{M}_{\mathrm{q}}^{\prime \prime}=\mathrm{m}_{\rho} / 2$ & NRCQM & $\mathrm{M}_{\mathrm{q}}=441$ \\
\hline & 3 & & Particle masses & & $\mathrm{M}_{\mathrm{q}}^{\omega}=\mathrm{m}_{\omega} / 2$ & Wick - Sternheimer & $\mathrm{n}_{\Sigma}-\mathrm{m}_{\mathrm{N}}-\mathrm{m}_{K}=441$ \\
\hline \multirow{2}{*}{$\begin{array}{c}1 \\
\mathrm{keV}\end{array}$} & 1 & & Particle masses & & & $\mathrm{N} \delta-\mathrm{m}_{\mathrm{n}}-\mathrm{m}_{\mathrm{e}}=161.6(1)$ & $170=\mathrm{m}_{\mathrm{e}} / 3$ \\
\hline & 8,3 & & & & & $\delta m_{N}=1293.3 \mathrm{keV}$ & $m_{e}=511.0 \mathrm{keV}$ \\
\hline \multirow{3}{*}{$\begin{array}{c}1 \\
\mathrm{keV}\end{array}$} & 1 & $9.5=\delta^{\prime}=8 \varepsilon^{\prime}$ & Nuclear data & 132 & & $161=\Delta^{T F}(\mathrm{Sb})$ & $170,168(\mathrm{Sn})$ \\
\hline & 2 & 19 & & 266 & & $322(\mathrm{Sb})$ & $339, \varepsilon_{n 2 n}$ \\
\hline & $3-6$ & $28-56$ & 492 (M.Ohkubo) & 533 & 606 & $644-962(\mathrm{Sb})$ & $511=\varepsilon_{o} / 2(\mathrm{Co})$ \\
\hline 2 & 1 & $11=\delta^{\prime \prime}=8 \varepsilon^{\prime \prime}$ & & & 176 & $187(\mathrm{Nd})$ & $\mathrm{D}$ in neutron \\
\hline $\mathrm{eV}$ & $4-8$ & 44 & $570(\mathrm{Sb})$ & & & $749,1500(\mathrm{Sb}, \mathrm{Pd}, \mathrm{Rh})$ & resonances \\
\hline
\end{tabular}




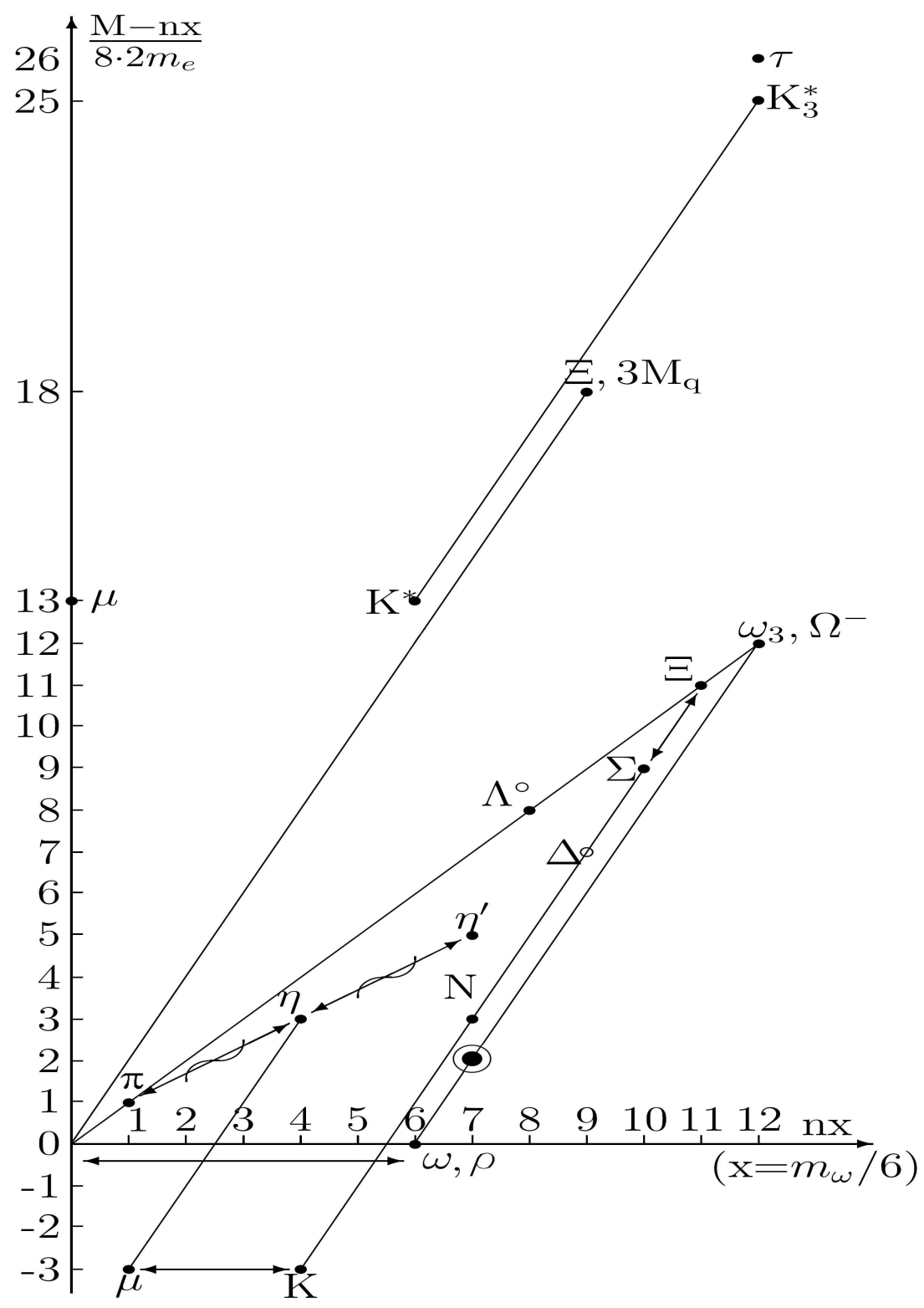

Figure 1: Position of the nucleon mass $\mathrm{N}$ among different mass intervals and particle masses in twodimensional presentation. Values on the horizontal axis are in units $16 \cdot 16 m_{e}$ coinciding with the pion parameter $f_{\pi}=130.7(4) \mathrm{MeV}$ close to $m_{\omega} / 6 \approx m_{\rho} / 6$. The remainder - values $M_{i}-\mathrm{k} \delta=M_{i}-\mathrm{k} 16 \cdot 16 m_{e}-$ are displayed along the vertical axis in units $16 m_{e}$. Three lines with different slopes correspond to:

1) the main parameter $\Delta M_{\Delta}=147 \mathrm{MeV}$ of baryon constituent quark masses from the nucleon $\Delta$-excitation $\left(m_{\Delta^{o}}-m_{n}\right) / 2=18 \delta$ and initial constituent quark mass $M_{q}=3 \times 147 \mathrm{MeV}$; initial baryon mass $3 M_{q}=m_{\Xi}$ (top);

2) the pion mass $140 \mathrm{MeV}=f_{\pi}+\delta(\mathrm{n}=16+1)$, masses of $\Lambda, \Xi$ and $\Omega$ hyperons are close to $8 m_{\pi}, 11 m_{\pi}, 12 m_{\pi}$ 3) stable interval in pseudoscalar meson $m_{\eta^{\prime}}-m_{\eta}=m_{\eta}-m_{\pi}^{ \pm}$(crossed arrows, $\mathrm{n}=50$ in units $\delta=16 m_{e}$. Nucleon $\Delta$-excitation and two s-quark masses $m_{s}$ result in a long line from the kaon $(\mathrm{K}, \mathrm{n}=4 \cdot 16-3=61)$ and the neutron mass $(\mathrm{N}, \mathrm{n}=7 \cdot 16+3=115)$ to the $\Xi^{-}$hyperon mass $(\Xi)$.

Nucleon mass in nuclear medium ( $m_{N}^{\text {nucl }}$, circled large point) is close to the sum of $\Delta M_{\Delta}=147 \mathrm{MeV}$ and $6 f_{\pi}=6 \cdot 16 \cdot 16 m_{e} \approx m_{\omega} \approx m_{\rho}$. It is the last member of the sequence of the baryon mass transformation from the initial mass $3 M_{q}=9 \Delta M_{\Delta}$ (at top, $\mathrm{n}=9 \cdot 18$ ) to $m_{N}^{\text {nucl }}$ at the bottom.

The mass of $\tau$ lepton and $2^{+}$excitation of vector mesons (shown at top) are considered elsewhere. They are close to integer numbers of the $M_{q} \approx 3 \Delta M_{\Delta}=(\alpha / 2 \pi) M_{H}$. 


\section{CODATA relation in the tuning effect}

Nucleon mass is a result of constituent quark-mass evolution: First, a reduction of each quark mass from $M_{q}(\mathrm{n}=3 \cdot 18)$ to $\Delta M_{\Delta}+2 f_{\pi}(\mathrm{n}=18+2 \cdot 16$ about 50-51). In Fig.1 it corresponds to the shift downwards from $3 M_{q}$ to $\Delta$. At the second step, due to spin-dependent interaction between quarks, nucleon mass looses $2 \Delta M_{\Delta}(\mathrm{n}=151-36=115)$. Nucleon mass in the nuclear medium $m_{N}^{\text {nucl }}$ (circled dot in Fig.1, about $8 \mathrm{MeV} \approx \delta$ below $m_{N}$ ) is shifted due to residual quark interaction. The generation of the initial mass and its reduction is explained in QCD and NRCQM [15-17]. The value $m_{N}^{\text {nucl }}$ is the last in a sequence from the initial mass $3 M_{q}=9 \Delta M_{\Delta}-$ to the $\Delta$-baryon mass $1320 \mathrm{MeV}=3\left(2 f_{\pi}+\Delta M_{\Delta}\right)=3 M_{q}^{\Delta}-$ to $m_{N}-$ and finally to $6 f_{\pi}+\Delta M_{\Delta}$. So, number $\mathrm{n}=115$ corresponds to the neutron mass.

The shift of the neutron mass relative to the value $115 \delta$ equal to $\delta m_{n}=161.65(6) \mathrm{keV}$ was derived from the recent precise ratio between neutron and electron masses evaluated by CODATA [18] $m_{n} / m_{e}=1838.6836605(1)$. The shift accounts an integer ratio with nucleon mass splitting $\delta m_{N}=1293.3 \mathrm{keV} \delta m_{N} / \delta m_{n}=8(1.0001(1))$. Such unexpected relation in CODATA results

$$
\begin{gathered}
m_{n}=115 \cdot 16 m_{e}-m_{e}-\delta m_{N} / 8 \\
m_{p}=115 \cdot 16 m_{e}-m_{e}-9 \delta m_{N} / 8
\end{gathered}
$$

allows conclusions concerning the general properties of the tunung effect in particle masses.

First of all, there are other mass parameters with integer representation of values with the period $\delta$ (three top lines in Table 2 [9]). Muon mass with the lepton ratio $\mathrm{L}=m_{\mu} / m_{e}=13 \cdot 16-1=207$ has the same combination of $\mathrm{n} \delta-m_{e}$ as the neutron mass. Other examples - values $f_{\pi}$ and $\Delta M_{\Delta}$.

Secondly, constituent quark masses $\left(M_{q}\right.$ and $M_{q}^{\prime \prime}$, Table 2$)$ which are produced with the gluonquark-dressing effect $[15,19]$ are in the lepton ratios $\mathrm{L}=207$ with the vector boson masses, namely, $L^{Z}=M_{Z} / M_{q}=207.4$ and $L^{W}=M_{W} / M_{q}^{\prime \prime}=207.5$.

Table 2. Comparison of particle masses with the period $16 \mathrm{~m}_{\mathrm{e}}=\delta=8176 \mathrm{keV}$ (comments - in $\mathrm{MeV}$, constant shift $\Delta=9 m_{e}$ is boxed).

\begin{tabular}{cccccc}
\hline Particle & $\mathrm{m}_{\mathrm{i}}, \mathrm{MeV}$ & $\mathrm{k}$ & $\mathrm{m}_{\mathrm{i}}-\mathrm{k} \cdot 16 \mathrm{~m}_{\mathrm{e}}$ & Diff. & Comments $(\mathrm{MeV})$ \\
\hline$\mu$ & $105.658367(4)$ & 13 & -0.6294 & $-m_{e}-0.118$ & \\
$f_{\pi}$ & $130.7(4)[20]$ & 16 & & $\approx 0$ & \\
$\Delta^{\circ}-\mathrm{n}$ & $294.2(2)$ & 36 & $\approx 0$ & $2\left(\Delta M_{\Delta}=147.1\right), \Delta E_{B}=147.2[4]$ \\
$M_{q} \mathrm{NRCQM}, m_{X i^{-}} / 3$ & 441 & $3 \cdot 18$ & & $\Delta E_{B}=441[4]$ \\
$M_{H} / 18 \cdot 16$ & 436 & $3 \cdot 18-\Delta$ & $5=-\Delta$ & $m_{d} \approx \Delta$ \\
$M_{Z} / \mathrm{L}$ & 440.5 & $3 \cdot 18$ & $\approx-2 m_{e}$ & \\
\hline$\eta^{\prime}-\eta, \eta-\pi^{ \pm}$ & 409 & 50 & $\approx 0$ & $\Delta E_{B}=409[4]$ \\
\cline { 1 - 4 }$M_{q}^{\prime \prime} \mathrm{NRCQM}, m_{\rho} / 2$ & 387.7 & 48 & $-4.60=-\Delta$ & $m_{d} \approx \Delta, m_{\rho}=775.49(34)[14]$ \\
$M_{W} / \mathrm{L}$ & 388.4 & $3 \cdot 16$ & $\approx-2 m_{e}$ & $3 f_{\pi}$ \\
\hline $\mathrm{n}$ & $939.5654(1)$ & $115-0.6726(1)$ & & $-\mathrm{m}_{\mathrm{e}}-(1 / 8) \delta \mathrm{m}_{\mathrm{N}}$ \\
$\mathrm{p}$ & $938.2720(1)$ & $115-1.9666(1)$ & & $-\mathrm{m}_{\mathrm{e}}-(9 / 8) \delta \mathrm{m}_{\mathrm{N}}$ \\
$\Sigma^{\circ}$ & $1192.64(2)$ & 146 & $-1.05(2)$ & & $-2 m_{e}=-1.022$ \\
$\Xi^{\circ}$ & $1314.86(20)$ & 161 & $-1.47(20)$ & & $-3 m_{e}=-1.533$ \\
\hline
\end{tabular}




\section{Empirical relations in SM-parameters}

Exactness of CODATA integer relation could mean the presence of the common general origin of all SM-interactions. It was R.Feynman who draw attention to the common vector character of all interactions [21]. He suggested that in the future "super-duper model" an understanding of the main parameters like $\alpha$ could be achieved. Relations with factor $\alpha=1 / 137$ are shown in Table 1 (boxed values related as $\alpha / 2 \pi$ ). The proximity of $\alpha / 2 \pi=115.9 \cdot 10^{-5}$ to the ratio between the well-know main SM-parameters - masses of the $\mu$-lepton and Z-boson $m_{\mu} / M_{Z}=115.9 \cdot 10^{-5}$ was noticed long ago. Similar relations with QED parameter for a short $\left(1 / M_{Z}\right)$ distance $\alpha_{Z}=1 / 129$ can be added: the ratio between $m_{e}$ and the pion mass $170 \mathrm{keV} / 140 \mathrm{MeV}=121 \cdot 10^{-5}$ and between the pion mass and unconfirmed mass $M_{H}^{\prime}=116 \mathrm{GeV}$ of the possible scalar boson (twice the mass of the groping effect at $58 \mathrm{GeV}$ discussed by S.Ting [22]), namely, $140 \mathrm{MeV} / 116 \mathrm{GeV}=121 \cdot 10^{-5}$, are close to $\alpha_{Z} / 2 \pi=123 \cdot 10^{-5}$. It was suggested by V.Belokurov and D.Shirkov [23] that the small QED factor $(\alpha / 2 \pi)$ could be found in the electron rest mass itself. We briefly consider here four possible connection of the obtained unexpected CODATA relations with other empirical observations.

1) Integer ratios $1: 16$ and $1 ; 9$ in CODATA relations $\left(m_{e}: \delta\right.$ and $\left.161 \mathrm{keV}: \delta m_{N}\right)$ as well as the ratio $1: 9$ in charge splitting $\left(m_{e}: \delta m_{\pi}\right)$ could be considered together with the recent quark masses estimations $m_{d}=4.78(9) \mathrm{MeV}$ close to $\Delta=9 m_{e}=4.60 \mathrm{MeV}$ and $m_{b}=4.18$ (1) $\mathrm{GeV}$ close to $9 M_{q}=4.0 \mathrm{GeV}[9,14]$ (if values would be confirmed). Observed integer relations in lepton masses $m_{\mu} / m_{e}=\mathrm{L}=13 \cdot 16-1$ coinciding with the similar ratio between masses of Z-bozon and the constituent quark $M_{q}$ were considered [9] in connection with the parity nonconsevation as the result of a possible symmetry motivated properties of fermions (currents) composed the mass. It could mean that masses $m_{e}$ and $M_{q}=m_{e}(\alpha / 2 \pi)^{-1}$ might be considered together with the rational relation $3 \cdot 18 \cdot 16 m_{e}$ for the large mass. Difference between these two large values accounts about $m_{e}$. We need theoretical understanding of the observed tuning effect supplemented with CODATA relation. The general trend in QCD-development based on lattice-calculations should be combined with symmetry properties of common components.

2) Indirect confirmation of the tuning effect in particle masses was obtained from analysis of excitations of nuclei where tensor phrases connected with one-meson exchange play the dominant role. Development of nuclear model where nucleon structure would be directly included should take into account existing results from analysis of nuclear binding energies. Here in cluster effects with systems like ${ }^{4} \mathrm{He}$ and ${ }^{6} \mathrm{He}$ parameters of the grouping of difference of binding energies $\Delta E_{B}$ clearly demonstrate the distinguished role of intervals connected with integer values of the electron mass $m_{e}$ (for example, $\Delta=9 m_{e}$ etc.). In Table 2 values $\Delta E_{B}=147.2 \mathrm{MeV}=32 \Delta$ coinciding with $\Delta M_{\Delta}$, $\Delta E_{B}=441 \mathrm{MeV}$ close to $3 \Delta M_{\Delta}, 409 \mathrm{MeV}$ close to $M_{q}^{\Delta}$ are given in comments.

3) Observed additional shift in masses of strange hyperons $\Sigma^{\circ}$ and $\Xi^{\circ}$ (possibly correlated with the strangeness, boxed in two bottom lines of Table 2) could be connected with transformations into the mass of the constituent quark. This effect could be important for understanding of the origin of the dark matter and possible estimation of the gravitation intercity considered in [24]).

4) Rational relations with the masses of heavy fermions $\left(M_{q}, M_{q}^{\prime \prime}\right)$ were extended to the scalar boon masses [9,11] (see the fifth line in Table 2). We expect theoretical interpretation of observed empirical effects. Some relations in masses of heavy bosons and quarks were discussed in $[9,11]$. 


\section{Conclusions}

Combined analysis of particle mass spectrum and nuclear data, considered both as results of quark-structure hadronization process, shows a distinguished role of values of charge mass splitting of the nucleon, the pion and leptons. Rational relations in particle masses including the lepton ratio could be considered as the manifestation of symmetry properties of the common fundamental components directly connected with the electron rest mass and properties of the physical condensate. Presence of CODATA relation, if confirmed, demands drastic modification of property of SM-condensate. Frank Wilczek named the electron rest mass "theoretical puzzle". If the empirical CODATA relation will be confirmed and commonly accepted, it will demonstrate an important role of the electron mass and the QED in the SM-development.

\section{References}

[1] J.P. Schiffer et al., Phys. Rev. Lett. 92 (2004) 162501, and private communication.

[2] T. Otsuka, T. Suzuki, Y. Utsuno, Y., Nucl. Phys. A 805 (2008) 127c.

[3] I. Tanihata, Mod. Phys. Lett. A 25 (2010) 1886, and private communication.

[4] S.I. Sukhoruchkin et al., Proc. ISINN-19. JINR E3-2012-30, pp. 284, 296, 308.

[5] S.I. Sukhoruchkin et al., Proc. ISINN-20, JINR E3-2013-22, p. 254.

[6] S.I. Sukhoruchkin et al., Proc. ISINN-21. JINR E3-2014-13, p. 255.

[7] S.I. Sukhoruchkin et al., Proc. ISINN-22. JINR E3-2015-30, pp. 265, 277, 289, 301.

[8] L-B New Series Vols. I/25A,E,H. Springer, 2012, 2013, 2015; H.Schopper (Ed.).

[9] S.I. Sukhoruchkin, Nucl. Part. Phys. Proc. (submit.).

[10] S.I. Sukhoruchkin, Stat. Properties of Nuclei. G.Garg (Ed.), p. 215. (Pl. Press, 1972).

[11] S.I. Sukhoruchkin, Nucl. Part. Phys. Proc. 258-259 (2015) 268.

[12] S.I. Sukhoruchkin, Nucl. Phys. B (Proc. Suppl.) 234 (2013) 241.

[13] S.I. Sukhoruchkin, J. Phys.: Conf. Ser. 447 (2013) 012056

[14] K.A.Olive et al. (Partice Data Group), Chin.Phys. C.2014, 38(9),090001.

[15] H.L.L. Roberts et al, Phys. Rev. C 83 (2011) 065206.

[16] L. Glozman, Nucl. Phys. A 629 (1998) 121c.

[17] C. Itoh et. al., Phys. Rev. D 40 (1989) 3660.

[18] P. Mohr et al., Rev. Mod. Phys. 84 (2012) 1527.

[19] L-B New Series Vol. I/22AB, Springer, 2009. H. Schopper (Ed.).

[20] M. Suzuki, J. Phys. G: Part. Nucl. Phys. 33 (2006) 535.

[21] R. Feynman, QED-Strange Theory of Light and Maatter, Princ. Univ. Press. 1986.

[22] S. Ting, CERN-PPE/93-34, 1993.

[23] V.V. Belokurov, D.V. Shirkov, The Theory of Particle Interactions. AIP, N.-Y., 1991.

[24] S.I. Sukhoruchkin, Program and Thesis, Proc. 40th Ann. Conf. Nucl. Spectrosc. Struct. At. Nucl., Leningrad, 1990, p. 147.

[25] S.I. Sukhoruchkin, Proc. XVI Workshop on High Energy Spin Phys., Dubna, 2015 (submit.). 